\title{
Lipid Trafficking at Membrane Contact Sites During Plant Development and Stress Response
}

\author{
Morgane Michaud* and Juliette Jouhet \\ UMR 5168 CNRS, CEA, INRA, Laboratoire de Physiologie Cellulaire et Végétale, Université Grenoble Alpes, \\ Grenoble, France
}

OPEN ACCESS

Edited by:

Yohann Boutté,

UMR 5200 Laboratoire de Biogenèse Membranaire (LBM), France

Reviewed by:

Christoph Benning,

Michigan State University,

United States

Abel Rosado,

University of British Columbia

Canada

*Correspondence:

Morgane Michaud

morgane.michaud@cea.fr

Specialty section:

This article was submitted to

Plant Cell Biology,

a section of the journal

Frontiers in Plant Science

Received: 07 November 2018

Accepted: 03 January 2019

Published: 15 January 2019

Citation:

Michaud M and Jouhet J (2019) Lipid Trafficking at Membrane Contact Sites During Plant Development and Stress Response. Front. Plant Sci. 10:2. doi: 10.3389/fpls.2019.00002
The biogenesis of cellular membranes involves an important traffic of lipids from their site of synthesis to their final destination. Lipid transfer can be mediated by vesicular or non-vesicular pathways. The non-vesicular pathway requires the close apposition of two membranes to form a functional platform, called membrane contact sites (MCSs), where lipids are exchanged. These last decades, MCSs have been observed between virtually all organelles and a role in lipid transfer has been demonstrated for some of them. In plants, the lipid composition of membranes is highly dynamic and can be drastically modified in response to environmental changes. This highlights the importance of understanding the mechanisms involved in the regulation of membrane lipid homeostasis in plants. This review summarizes our current knowledge about the non-vesicular transport of lipids at MCSs in plants and its regulation during stress.

Keywords: lipid transfer, membrane contact sites, higher plants, stress, membrane homeostasis

\section{INTRODUCTION}

Cellular membranes are composed of a specific assembly of lipids and proteins defining their functions and identities. Different types of lipids are found in membranes, the most abundant being glycerolipids present in all membranes, then sterols, and sphingolipids particularly enriched in the PM (Li-Beisson et al., 2013). Glycerolipids are composed of a glycerol backbone esterified with two FAs and of a polar head, which composition defines different families, such as the phospholipids and the galactoglycerolipids. A third FA can also be esterified on the glycerol backbone to form the TAGs, which are reserve lipids stored in LDs. In plants, FAs are synthesized in plastids and a fraction is exported to fulfill the synthesis of glycerolipids in extra-plastidial membranes (Li-Beisson et al., 2013, 2017). Phospholipids contain a Pi group in their polar head. They are mainly synthesized in the ER from PA and constitute the major glycerolipids present within extraplastidial membranes (Figure 1; Li-Beisson et al., 2013; Michaud et al., 2017). Galactoglycerolipids do not contain a Pi group but one or two galactose residues to form the MGDG and DGDG, respectively. These lipids are synthesized in plastids and are mainly located in this compartment under normal growth conditions (Boudiere et al., 2012). The biosynthesis of galactoglycerolipids inside chloroplasts relies on precursor lipids synthesized in the ER, by the so-called eukaryotic pathway, and in some plant species, from precursors synthesized directly in plastids by the

Abbreviations: DAG, diacylglycerol; DGD, digalactosyldiacylglycerol synthase; DGDG, digalactosyldiacylglycerol; ER, endoplasmic reticulum; E-Syt, extended-synaptotagmin; FA, fatty acid; IE, inner envelope of plastids; LD, lipid droplet; LTP, lipid transfer protein; MCS, membrane contact site; MGD, monogalactosyldiacylglycerol synthase; MGDG, monogalactosyldiacylglycerol; MTL, mitochondrial transmembrane lipoprotein complex; MTX, methotrexate; OE, outer envelope of plastids; ORP, oxysterol binding protein (OSBP) related protein; PA, phosphatidic acid; PAM, plasma membrane associated membrane; PBM, peribacteroid membrane; PC, phosphatidylcholine; Pi, phosphate; PLAM, plastid associated membrane; PM, plasma membrane; TAG, triacylglycerol; VAP, vesicle-associated membrane protein (VAMP)-associated protein. 
prokaryotic pathway (Mongrand et al., 1998; Block and Jouhet, 2015; Mueller-Schuessele and Michaud, 2018). Under certain stresses, like Pi starvation, DGDG is also found in extra-plastidial membranes (Hartel et al., 2000; Dormann and Benning, 2002; LaBrant et al., 2018). Thus, because lipids are synthesized at specific locations in the cell and have then to be distributed to other cell compartments, an important and complex traffic of lipids between membranes is required to sustain organelle biogenesis in cells.

Lipid transfer between organelles can be mediated by vesicular or non-vesicular pathways. Some compartments, such as the Golgi and the PM, import lipids via these two pathways (Jurgens, 2004). Some other organelles, like mitochondria or plastids, rely mostly on non-vesicular routes for the biogenesis of their membranes (Figure 1; (Hurlock et al., 2014; Block and Jouhet, 2015; Michaud et al., 2017). Non-vesicular lipid transfer requires, (1) the desorption of the lipid from the donor membrane, which is the limiting step of the process, (2) the movement of this hydrophobic molecule across a soluble phase, and (3) its insertion into the acceptor membrane. The spontaneous transfer of glycerolipids is a very long process that has been judged irrelevant in vivo (Phillips et al., 1987). Some proteins, called LTPs, facilitate non-vesicular lipid transfer. LTPs are composed of a hydrophobic pocket accommodating lipids and stimulate the steps 1 and 2 of the transfer process (Wong et al., 2017). Lipid exchange is thought to occur at MCSs between organelles. MCSs are regions where two membranes are at a distance of less than $30 \mathrm{~nm}$ and form functional platforms (Phillips and Voeltz, 2016;

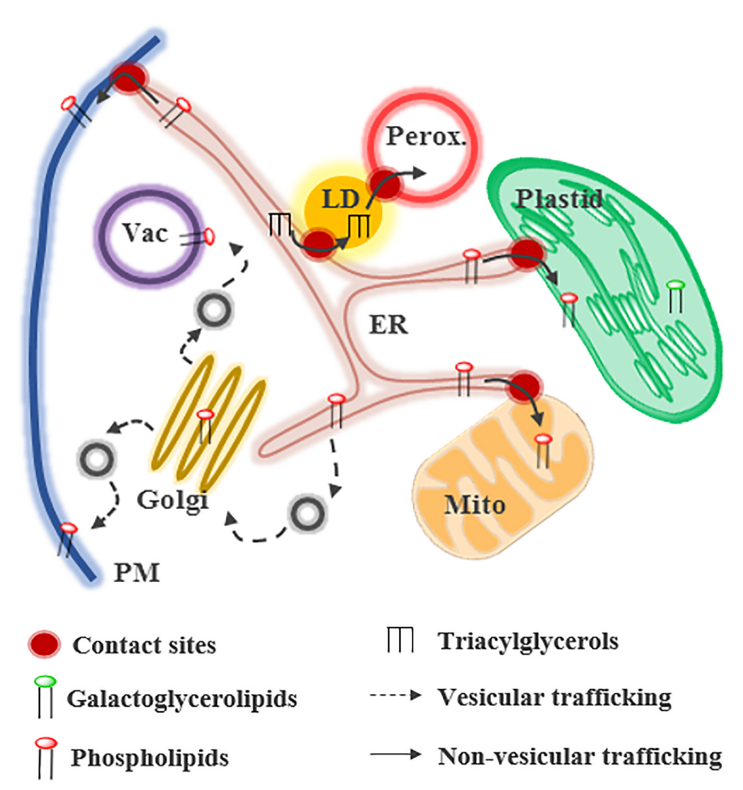

FIGURE 1 | Glycerolipid trafficking in plant cells. Lipids can be transferred by vesicular or non-vesicular routes at contact sites between membranes. Phospholipids and triacylglycerols are mainly synthesized in the endoplasmic reticulum (ER) whereas galactoglycerolipids are synthesized in plastids. In presence of phosphate $(\mathrm{Pi})$, galactoglycerolipids are located primary in plastids and extra-plastidial membranes contain phospholipids. LD, lipid droplet; Mito, mitochondria; Perox, peroxisome.
Wu et al., 2018). They are formed and maintained by tethering proteins, which bridge the two membranes. Even if MCSs have been observed between almost all organelles in many species (Perez-Sancho et al., 2016; Cohen et al., 2018), their composition and the mechanisms involved in lipid transfer are still largely unknown, particularly in plants. This review summarizes our current knowledge about glycerolipid transfer at MCSs in plants and the regulation of this process in response to stress.

\section{LIPID TRAFFICKING DURING PLANT DEVELOPMENT}

\section{Lipid Trafficking at ER-Plastids MCSs}

Lipid transport from ER to plastids is thought to occur by nonvesicular transfer at MCSs (Hurlock et al., 2014; Block and Jouhet, 2015; LaBrant et al., 2018; Mueller-Schuessele and Michaud, 2018). ER-plastids MCSs have been observed by microscopy and a fraction of the ER is tightly attached to purified chloroplasts (Renaudin and Capdepon, 1977; Andersson et al., 2007; Schattat et al., 2011). This fraction, called PLAM, has a particular protein and lipid composition and contains a PC synthase activity, supporting a role of ER-plastids MCSs in lipid metabolism (Andersson et al., 2007). Plastids are able to form protrusions of around $1 \mu \mathrm{m}$ of diameter called stromules. Stromules are highly dynamic structures that are regulated during plant development or in response to different biotic or abiotic stresses (Kohler and Hanson, 2000; Hanson and Sattarzadeh, 2011; Krenz et al., 2014). They are able to interact with other cell compartments, including the ER (Schattat et al., 2011). However, the role of stromuleorganelle interactions in lipid exchanges is still unknown and remains to be investigated.

It is well known that PC synthesized in the ER is the main precursor for galactoglycerolipids synthesis in plastids (Andersson et al., 2004; Benning, 2008). MGDG synthesis is mainly catalyzed by the MGDG synthase 1 (MGD1), located in the inner envelope (IE) of plastids. Then, DGD synthases (DGD) located in the plastid OE synthesize DGDG from MGDG. MGD1 uses diacylglycerol (DAG) as a substrate, meaning that DAG, generated from PC synthesized in the ER, has to be located in the IE. It is not clear where the DAG required for this process is initially generated and there are still debates concerning which lipid(s) are translocated from the ER to plastids. Currently, PC, lyso-PC (i.e., a PC with one FA), PA and/or DAG have been proposed to be the transported molecules. It is likely that several mechanisms involved in the transport of different lipids co-exist in cells to ensure the biogenesis of plastid membranes in different conditions.

Isolated plastids are able to uptake PC, but not PE, coming from microsomes or liposomes (i.e., small vesicles mimicking membranes), supporting the transfer of PC molecules from the ER to plastids (Andersson et al., 2004; Yin et al., 2015). In addition, a lipid flippase, ALA10, able to translocate phospholipids from one leaflet of a membrane to another, was reported to stimulate PC export from the ER in Arabidopsis thaliana (Poulsen et al., 2015; Botella et al., 2016). When ALA10 interacts with ALIS5, the protein is located in the ER in close 
proximity to plastids (Botella et al., 2016; Figure 2). ALA10 stimulates leaf growth and increases the ratio of MGDG/PC, suggesting that MGDG synthesis from PC via the eukaryotic pathway is stimulated by this protein. The authors proposed that ALA10 might create a specific lipid environment in the ER favoring lipid transfer by flipping specific PC species from the luminal to the cytosolic leaflet of the ER (Botella et al., 2016). Once in the plastid envelope, PC might be degraded by phospholipases to form PA, which activates MGD1 and promotes galactoglycerolipids and plastid membranes biogenesis (Dubots et al., 2010; Botella et al., 2016).

The transport of lyso-PC from the ER to plastids seems an advantageous model as lyso-PC molecules are soluble enough to freely diffuse through the cytosol (Somerharju, 2015). This transfer is supported by pulse-chase experiments following the import of ER-synthesized lipids in leek plastids (Mongrand et al., 2000). In addition, the presence of enzymes required to generate lyso-PC in the ER and to synthesize PC by acylation after transfer in the OE of plastids have been demonstrated (Figure 2). Indeed, a lipase (BnClip1), involved in plastid development, is located at ER-plastids MCSs in Brassica napus (Tan et al., 2011) and might generate lyso-PC. Two long-chain acyl-CoA synthases, LACS7 and LACS9 located in the ER and plastids, respectively, were shown to influence the eukaryotic pathway of plastid lipids synthesis in A. thaliana (Jessen et al., 2015). It was proposed that these enzymes synthesize a specific pool of acyl-CoA at ERplastids MCSs to sustain galactoglycerolipids synthesis (Figure 2; Jessen et al., 2015). Finally, a lyso-PC acyltransferase (LPCAT) activity was detected in leek plastid envelope (Bessoule et al., 1995).

The transfer of PA to plastids was suggested because two subunits of a complex transporting lipid precursors via the eukaryotic pathway, the TGD (Trigalactosyldiacylglycerol) complex, are able to bind PA (Awai et al., 2006; Wang et al., 2012). The composition and role of the TGD complex in plastid membranes biogenesis have been recently reviewed (Hurlock et al., 2014; LaBrant et al., 2018; Mueller-Schuessele and Michaud,
2018). The TGD complex is located at the interface between the $\mathrm{OE}$ and the IE (Figure 2) and is composed of five subunits, three of them (TGD1, 2, and 3) forming an ABC transporter in the IE, TGD4 being in the OE and TGD5 bridging the OE subunit TGD4 with the TGD1, 2, 3 complex in the IE. This complex transfers lipids from the OE to the IE to feed the MGD1 enzyme with precursors coming from the eukaryotic pathway. Whether it also transfers lipids from the ER to the OE and the nature of the transported lipid(s) are still unknown. TGD2 and TGD4 are able to bind PA (Awai et al., 2006; Wang et al., 2012), but this binding might reflect a regulatory role of PA, like for MGD1, rather than a PA transport activity. Recently, it has been suggested that DGD1 could also be involved in the transfer of lipids between the OE and the IE of plastids (Kelly et al., 2016). The N-terminal extension (NDGD1) of DGD1, required for DGD1 insertion in the OE, is essential for the synthesis of DGDG in the OE (Figure 2; Kelly et al., 2016). In vitro, purified NDGD1 is able to bind PA and triggers liposome fusion. It was proposed that NDGD1 mediates fusion or hemi-fusion of plastid envelopes by creating a PA-enriched environment, favoring the transport of galactoglycerolipids and/or precursors between both membranes (Kelly et al., 2016). TGD2 is also able to destabilize liposomes in vitro (Roston et al., 2011), thus it would be of interest to investigate whether the TGD complex and the $\mathrm{N}$-terminal extension of DGD1 act in concert or not to modulate membrane structures and to favor lipid transfer between $\mathrm{OE}$ and IE.

The role of DAG in the regulation of galactoglycerolipids synthesis is supported by a mathematical modeling of the lipid fluxes required between $\mathrm{OE}$ and IE to sustain membrane biogenesis (Marechal and Bastien, 2014). This model shows that, if PA was the main imported molecule, a huge, nonphysiologically relevant concentration of PA in the IE would be required to sustain plastid lipids synthesis (Marechal and Bastien, 2014). Thus, this model favors the import of DAG precursor and a role of PA in the regulation of the galactoglycerolipid synthesis pathways.

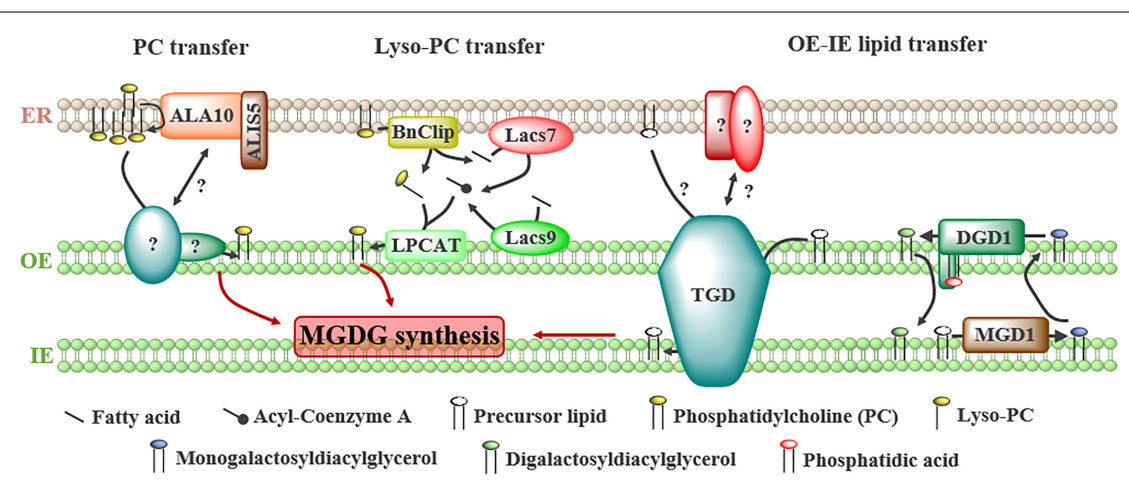

FIGURE 2 | Proteins involved in the transport of lipids from the ER to plastids and between the outer (OE) and the inner (IE) envelope of plastids to fulfill galactoglycerolipid synthesis. Several precursors have been proposed to be transported from ER: (1) phosphatidylcholine (PC), whose transfer is stimulated by the flippase ALA10 located at ER-plastids junctions by interacting with ALIS5, (2) lyso-PC, generated from PC by a lipase, BnClip1, and reacylated in the OE by a lyso-PC acyltransferase (LPCAT) from acyl-Coenzyme A synthesized at ER-plastids contact sites by the long-chain acyl-CoA synthases LACS7 and LACS9, (3) unknown precursors or galactolipids transported from the OE to the inner envelope (IE) by the trigalactosyldiacylglycerol (TGD) complex or the digalactosyldiacylglycerol (DGDG) synthase DGD1. 
According to the essential role of galactoglycerolipids in thylakoid biogenesis and photosynthesis, it is thought that lipid transfer from the ER is a crucial process that has to be tightly regulated during plant development. In accordance with this, TGD mutant plants present severe growth and developmental defects, such as defects in embryo and seed development, as well as impairments in photosynthesis, thylakoids development, and chloroplasts division (Xu et al., 2005; Fan and Xu, 2011; Li et al., 2012). According to transcriptomic data available on the Arabidopsis eFP browser (Winter et al., 2007), the TGD proteins are expressed in most of the plant tissues, supporting a role of this complex throughout plant development (Figure 3). Because of the different pathways that might be involved in plastids lipid transport (Figure 2), it would be of interest to investigate the contribution of each pathway during the different stages of plant development.

\section{Lipid Trafficking at ER-PM MCSs}

The non-vesicular transfer of lipids from the ER to the PM in plants is suggested by pulse chase experiments showing that the transfer of several phospholipids is reduced but not blocked when vesicular trafficking is inhibited (Moreau et al., 1998). However, whether this transfer occurs at ER-PM contact sites and the actors and mechanisms involved are still unknown. These last years, several proteins located at ER-PM junctions were identified in plants (Perez-Sancho et al., 2016; Bayer et al., 2017). By homology with what is known from yeast and mammalian cells, it is thought that ER-PM contact sites in plants would also be involved in lipid transfer and that some actors might be conserved [for review, see (Perez-Sancho et al., 2016; Chang et al., 2017; Wang et al., 2017; Saheki and De Camilli, 2017a)].

In peas, an ER fraction associated to the PM, called PAM (PM associated membrane), was isolated (Larsson et al., 2007). A LPCAT activity has been detected in PAM and in the PM, indicating that PC could be exchanged at ER-PM MCSs via the lyso-PC intermediate, as it has been suggested at ER-plastids MCSs (see above) (Larsson et al., 2007).

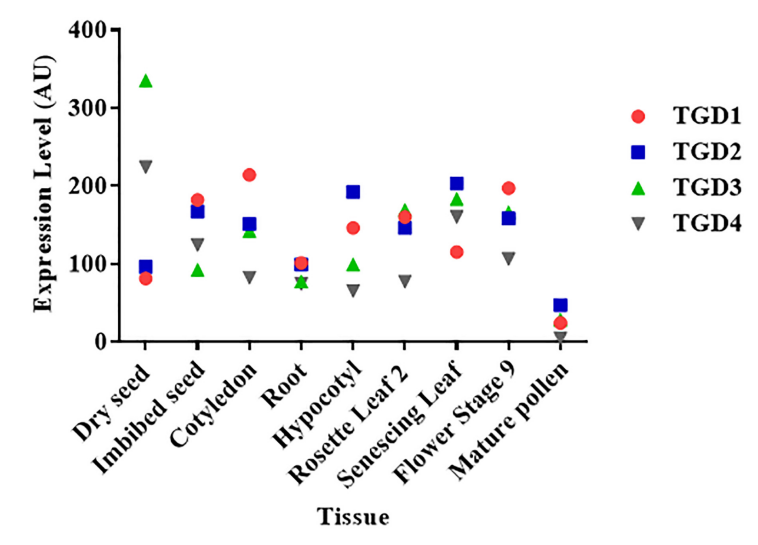

FIGURE 3 | Level of expression of the TGD complex subunits in different Arabidopsis thaliana plant tissues. The expression levels of each gene in arbitrary units $(\mathrm{AU})$ were retrieved from the Arabidopsis eFP browser (Winter et al., 2007). Data for the TGD5 subunit were not available.
Two families of ER proteins, the VAP and the E-Syt, involved in MCSs formation and lipid homeostasis in yeast and mammals, are conserved in plants (Perez-Sancho et al., 2016; Saheki and De Camilli, 2017a,b). VAP proteins interact with several LTPs, especially from the ORP family, at different MCSs (Murphy and Levine, 2016; Saheki and De Camilli, 2017a). In A. thaliana, three VAP homologs (VAP27-1, VAP27-3, and VAP27-4) have been located at ER-PM junctions and VAP27-3 interacts with ORP3a, a LTP able to bind and extract sitosterol in vitro (Saravanan et al., 2009; Wang et al., 2016). E-Syts are proteins tethering ER and PM in yeast and mammals and are able to transport lipids in vitro (Saheki and De Camilli, 2017b). In A. thaliana, AtSyt1 is a member of the E-Syt LTP family involved in the maintenance of PM integrity (Lewis and Lazarowitz, 2010) and endosome recycling (Lewis and Lazarowitz, 2010; Kim et al., 2016). AtSyt1 is located in the ER, shows specific enrichment at contact sites with PM and seems to be required for the maintenance of these junctions (Levy et al., 2015; Perez-Sancho et al., 2015; Siao et al., 2016). Despite its ability to bind in vitro liposomes and several phospholipids such as PA, phosphatidylinositolphosphates or phosphatidylserine (Schapire et al., 2008; PerezSancho et al., 2015), the direct role of AtSyt1 in lipid transport is still elusive. It might have an indirect role by regulating ER-PM apposition. The lipid-binding activity of AtSyt1 is mediated by the two $\mathrm{C} 2$ domains of the protein and can be $\mathrm{Ca}^{2+}$ dependent, suggesting that ER-PM MCSs might be regulated by $\mathrm{Ca}^{2+}$ signaling (Schapire et al., 2008). In addition, AtSyt1 is involved in plant responses to different stresses, including osmotic, salt, mechanical stresses or $\mathrm{Ca}^{2+}$-dependent freezing tolerance, showing the importance of this protein and of ER-PM MCSs in PM homeostasis during stress (Eckardt, 2008; Schapire et al., 2008; Yamazaki et al., 2008; Perez-Sancho et al., 2015). These first examples of proteins located at ER-PM junctions in plants support the involvement of such structures in lipid homeostasis. However, further investigations are required to identify the actors and regulatory mechanisms involved, particularly in stress conditions.

Analysis of ER-PM MCSs during plant development has revealed that these structures are present in all plant tissues, including, roots, hypocotyls and leaves, but are more abundant in young tissues and elongating cells, suggesting an important role of ER-PM MCSs during active PM biogenesis (McFarlane et al., 2017). The distance between the ER and the PM is $\leq 15 \mathrm{~nm}$ and ER-PM MCSs have an average length of $160 \mathrm{~nm}$. Interestingly, ER-PM MCSs are free of ribosomes but contain vesicles and microtubules, consistent with the importance of the cytoskeleton in the regulation of the localization of some ER-PM proteins, such as VAP27 (Wang et al., 2014; McFarlane et al., 2017). Overall, ER-PM MCSs seem to be important structures implicated in the regulation of $\mathrm{PM}$ homeostasis during both plant development and stress response.

\section{Lipid Trafficking at LDs-Organelle MCSs}

In plants, LDs are formed either in the chloroplast to form plastoglobuli or in the ER. Little is known about the mechanistic details in plants and most of the studies were performed in tissues that accumulate and degrade oil (i.e., seeds and pollen tubes) 
and therefore in ER derived LDs (Pyc et al., 2017). LDs are composed of a core of neutral lipids, mainly TAGs, surrounded by a monolayer of phospholipids. Synthesis of TAGs occurs in the ER (Figure 1) and then TAGs accumulate between the two leaflets of the ER membrane. To stabilize the ER-LD junction during the maturation of the LD, SEIPIN proteins are recruited and facilitate the incorporation of additional proteins and lipids into the growing LD (Cai et al., 2015; Salo et al., 2016). During this step, CCT1 (CTP:phosphocholine cytidyltransferase 1) is recruited to the $\mathrm{LD}$ surface to help the coordination of the monolayer synthesis with the expansion of the LD size (Krahmer et al., 2011). It is not known whether the accumulation of TAGs in LDs relies only on their synthesis at ER-LD MCSs, or directly on LDs as in mammals (Wilfling et al., 2013; Olzmann and Carvalho, 2018), or if specific proteins are required to transfer TAGs from the ER to the LDs. Once mature, the LDs can detach from the ER by unknown process and interact with other organelles, such as peroxisomes, mitochondria, chloroplasts or vacuoles (Gao and Goodman, 2015).

Lipid droplets and TAGs play an important role at different stages of plant development including germination, flower development or pollen tube growth (Huang, 2018; Yang and Benning, 2018). During seed germination, TAGs breakdown allows the synthesis of sucrose, which serves as the main source of energy. The hydrolysis of TAGs, catalyzed by the TAG lipase SDP1 (sugar dependent 1), releases FAs that are transported in peroxisomes by the $\mathrm{ABC}$ transporter PXA1 and are degraded by $\beta$-oxidation to produce acetylCoA (Hayashi et al., 2002; Eastmond, 2006). TAG lipolysis during germination involves the direct interaction of LDs with peroxisomes, which is regulated by sucrose availability (Cui et al., 2016). This interaction facilitates the traffic of the lipase SDP1 from the peroxisome to the LD via the retromer complex and the formation of peroxisome extensions (ThazarPoulot et al., 2015). PXA1 seems to regulate the extent of interactions between LDs and peroxisomes during germination (Cui et al., 2016). However, further investigation is required to understand whether or not PXA1 is directly involved in the establishment of LDs-peroxisomes MCSs for TAG breakdown during germination and to identify the other actors involved in such processes.

\section{LIPID TRAFFICKING UNDER STRESS CONDITION}

Many stresses, such as high light, cold or nutrient stresses, are known to induce a modification of the lipid composition of cellular membranes in plants (Sandelius and Liljenberg, 1982; Jouhet et al., 2003; Zheng et al., 2016). The most common and studied situation leading to a spectacular remodeling of membrane lipids is triggered by Pi starvation.

\section{Lipid Remodeling During Phosphate Starvation}

Phosphate starvation is a stress commonly encountered by plants that induces a wide variety of mechanisms to optimize
Pi uptake from soil and $\mathrm{Pi}$ remobilization from intracellular reserves. As phospholipids retain up to one third of cellular $\mathrm{Pi}$ in plant cells, they constitute a valuable source of $\mathrm{Pi}$ (Poirier et al., 1991). Thus, during Pi deficiency, phospholipids are partially degraded to release $\mathrm{Pi}$ and are replaced by DGDG (Hartel et al., 2001; Jouhet et al., 2003; Moellering and Benning, 2011). As DGDG is synthesized in plastids, a massive transfer to mitochondria, vacuoles and the PM was shown to occur in absence of Pi (Hartel et al., 2000; Jouhet et al., 2004; Andersson et al., 2005). The transfer of DGDG from plastids to mitochondria is thought to take place at MCSs, which increase in number in this condition (Figure 4). However, whether DGDG is transported to the PM and vacuoles by vesicular or non-vesicular routes remains unknown. The transport of DGDG from plastids to mitochondria and the export of phosphatidylethanolamine for degradation or recycling are partially mediated by the Mitochondrial Transmembrane Lipoprotein (MTL) complex (Figure 4; Michaud et al., 2016, 2017). This huge complex enriched in lipids is located at MCSs between mitochondrial membranes and might also contact other organelles. AtMic60, a conserved protein of the inner membrane, plays a role in the lipid transport process (1) by regulating the proximity between mitochondrial membranes via its interaction with the outer membrane protein Tom40 and (2) by destabilizing membranes, likely to promote lipid desorption (Figure 4; Michaud et al., 2016). As this complex is detected in +Pi and Pi conditions and most of the components are conserved during evolution, it might have a broader role in mitochondrial lipid trafficking.

Interestingly, analysis of the FA composition of DGDG molecules during $\mathrm{Pi}$ starvation has suggested that DAG backbones coming from degraded PC molecules could serve as a substrate for DGDG synthesis in plastids (Jouhet et al., 2003), raising questions about (1) the site(s) of phospholipids degradation and (2) the trafficking routes of the DAG backbones recycled from PC. Indeed, PC or PC-derivative lipids might be directly transported from mitochondria, $\mathrm{PM}$ and vacuoles to the plastids. Alternatively, PC molecules might be transported to the ER before being imported into plastids by the existing pathways to synthesize DGDG. The subcellular localization of several phospholipases, which are induced during Pi starvation and able to degrade phospholipid polar heads, suggests that phospholipids might be degraded at the surface of organelles. The vacuolar phospholipase D, PLD $\zeta 2$, is localized at vacuoles-mitochondria and vacuoles-plastids MCSs during $\mathrm{Pi}$ starvation, suggesting that PA can be formed at the surface of mitochondria and plastids (Yamaryo et al., 2008). In addition, the phospholipase C NPC5 is localized in the cytosol and might associate with different organelle membranes to form the DAG backbones required for DGDG synthesis during Pi starvation (Gaude et al., 2008). Recently, the protein LPTD1 was shown to play an important role in the synthesis of galactoglycerolipids during $\mathrm{Pi}$ starvation in A. thaliana (Hsueh et al., 2017). LPTD1 is located in the $\mathrm{OE}$ of plastids and is highly induced in the absence of $\mathrm{Pi}$ (Hsueh et al., 2017). Lptd1 mutant plants show an alteration 


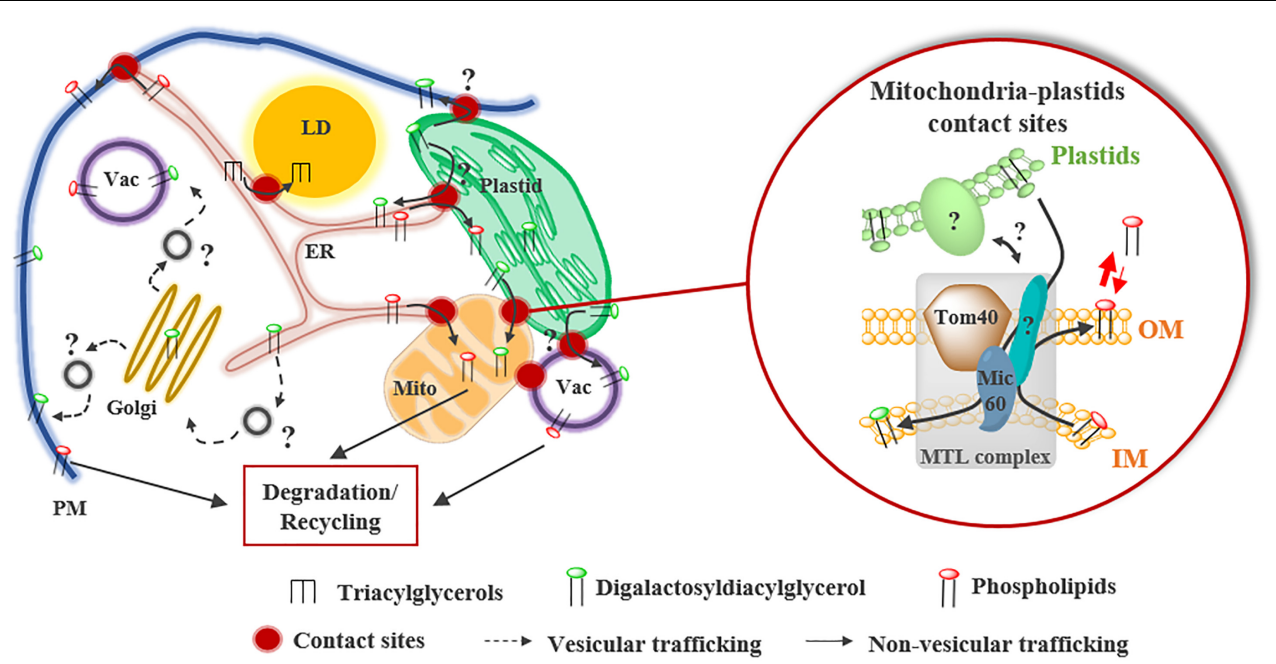

FIGURE 4 | Lipid remodeling during Pi starvation in higher plants. During Pi starvation, phospholipids are partially degraded and replaced by the galactoglycerolipid DGDG synthesized in plastids. DGDG transport from plastids to mitochondria (Mito) occurs at contact sites and is partly mediated by the mitochondrial transmembrane lipoprotein (MTL) complex. Inside this complex, Mic60, located in the inner membrane (IM) of mitochondria, plays a key role in the import of DGDG from plastids and in the export of phosphatidylethanolamine from mitochondria. Mic60 interacts with Tom40 located in the outer membrane (OM) of mitochondria at OM-IM contact sites. Whether the transfer of DGDG to plasma membrane (PM) and vacuole (Vac) occurs at contact sites or via the vesicular transfer pathway is still unknown.

of the lipid remodeling during Pi starvation, in particular, no increase of the DGDG content was observed in this mutant (Hsueh et al., 2017). Thus, LPTD1 could play a role in the recycling of phospholipid backbones in response to $\mathrm{Pi}$ starvation. As LPTD1 is a homolog of the TGD4 protein (Hsueh et al., 2017), it would be interesting to know whether LPTD1 is also part of the TGD complex and whether it is involved in lipid transfer from the ER and/or from other organelles to plastids to sustain phospholipids recycling in this situation.

\section{Symbiosis During Nitrogen Starvation}

In response to some nutrient stresses, especially nitrogen starvation, a wide variety of vascular plants establish a symbiotic relationship with specific soil microorganisms to favor nutrient uptake (Harrison and Ivanov, 2017). As an example, the symbiosis between legumes and soil nitrogen-fixing bacteria called rhizobia is crucial to cope with nitrogen-deficient environments. This symbiosis leads to the formation of a specific organ, the root nodule, where nutrient exchanges occur between the host and the bacteria (Udvardi and Poole, 2013; Downie, 2014). Rhizobia invade nodule cells by endocytosis, leading to the development of symbiosomes, an intracellular compartment where rhizobia divide and eventually differentiate into nitrogenfixing bacteroids (Udvardi and Poole, 2013). Symbiosomes are surrounded by a membrane, the PBM originating from the host cell, and can occupy a large part of the cytosol of infected cells (Udvardi and Poole, 2013; Downie, 2014). Thus, a tremendous amount of lipid synthesis and transport is required to sustain PBM synthesis and symbiosome development. The PBM seems to arise from the ER and the Golgi via the vesicular trafficking pathway (Whitehead and Day, 1997; Harrison and Ivanov,
2017). However, in soybean and Chinese vetch nodules, a significant amount of DGDG was detected in PBM, suggesting that plastids are also a source of lipids for PBM biogenesis (Gaude et al., 2004; Lei et al., 2014). More recently, different LTPs, AsE246 in Chinese milk vetch, and MtSyt1, MtSyt2, and MtSyt3 in Medicago truncatula, were shown to be involved in nodulation and development of symbiosomes (Lei et al., 2014; Gavrin et al., 2017). AsE246 is highly expressed in nodules, localized to the PBM and is able to bind several glycerolipids, such as PC and DGDG (Chou et al., 2006; Lei et al., 2014). MtSyt1, MtSyt2, and MtSyt3, which belong to the E-Syt LTP family, are highly abundant in infected nodule cells and are located to region of membrane expansion. In particular, MtSyt1 is abundant in the PBM and in the ER (Gavrin et al., 2017). These exciting results suggest that non-vesicular lipid trafficking could also be involved in the biogenesis of membranes required for the establishment of symbiosis between plant cells and microorganisms. However, further investigation is required to understand the function of LTPs and non-vesicular lipid transfer in membrane expansion during symbiosis and if lipid transfer occurs via MCSs between ER or plastids and PBM.

\section{Accumulation of Lipid Droplets During Stress}

Lipid droplets have a well-known function in energy storage but proteomic analyses of plant LDs showed that they might also play alternative roles, particularly during stress (Pyc et al., 2017; Yang and Benning, 2018). TAG accumulation was observed during nitrogen or Pi deprivation in higher plants (Gaude et al., 2007; Pant et al., 2015; Shimojima et al., 2015; Mei et al., 2017), and such increases were also significantly enhanced in 
the presence of sugar (Yang et al., 2011). In A. thaliana cell cultures, TAG accumulation seems to be a frequent response to cell growth arrest mediated by nutrient stresses or chemical drugs, such as MTX (Mei et al., 2017). In nitrogen starvation or MTX treatment, TAGs are synthesized from DAG backbones generated from PC and incorporated in LDs. However, whereas MTX treatment does not affect membrane lipids, their levels are significantly decreased during nitrogen deficiency, suggesting that membrane lipids are re-routed to synthesize reserve lipids in this condition (Mei et al., 2017). Thus, TAG accumulation seems to be mediated by different pathways depending on the stress condition. Currently, few information is available concerning the actors involved in LD biogenesis in response to different stresses. As expected, enzymes involved in TAG synthesis, such as the DAG acyltransferase DGAT1, are overexpressed in response to nitrogen starvation and MTX treatment (Mei et al., 2017). I would be of interest to know if proteins involved in ER-LD contacts and LD biogenesis, like SEIPINS, are also overexpressed or if specific stress-response proteins are required. In addition, further investigation is required to understand how membrane lipids are transported to sustain LD biogenesis and what role this organelle plays, in addition to lipid storage in plants.

\section{PERSPECTIVES}

The non-vesicular transfer of lipids at MCSs is a wide topic that has just started to be explored in plants. Plants are a particularly exciting model because of the wide variety of stresses triggering remodeling of MCSs and membrane lipid composition (Moellering and Benning, 2011; Mueller-Schuessele

\section{REFERENCES}

Andersson, M. X., Goksor, M., and Sandelius, A. S. (2007). Optical manipulation reveals strong attracting forces at membrane contact sites between endoplasmic reticulum and chloroplasts. J. Biol. Chem. 282, 1170-1174. doi: 10.1074/Jbc. M608124200

Andersson, M. X., Kjellberg, J. M., and Sandelius, A. S. (2004). The involvement of cytosolic lipases in converting phosphatidyl choline to substrate for galactolipid synthesis in the chloroplast envelope. Biochim. Biophys. Acta 1684, 46-53. doi: 10.1016/j.bbalip.2004.06.003

Andersson, M. X., Larsson, K. E., Tjellstrom, H., Liljenberg, C., and Sandelius, A. S. (2005). Phosphate-limited oat. The plasma membrane and the tonoplast as major targets for phospholipid-to-glycolipid replacement and stimulation of phospholipases in the plasma membrane. J. Biol. Chem. 280, 27578-27586. doi: 10.1074/jbc.M503273200

Awai, K., Xu, C., Tamot, B., and Benning, C. (2006). A phosphatidic acidbinding protein of the chloroplast inner envelope membrane involved in lipid trafficking. Proc. Natl. Acad. Sci. U.S.A. 103, 10817-10822. doi: 10.1073/pnas. 0602754103

Bayer, E. M., Sparkes, I., Vanneste, S., and Rosado, A. (2017). From shaping organelles to signalling platforms: the emerging functions of plant ER-PM contact sites. Curr. Opin. Plant Biol. 40, 89-96. doi: 10.1016/j.pbi.2017.08.006

Benning, C. (2008). A role for lipid trafficking in chloroplast biogenesis. Prog. Lipid Res. 47, 381-389. doi: 10.1016/j.plipres.2008.04.001

Bessoule, J. J., Testet, E., and Cassagne, C. (1995). Synthesis of phosphatidylcholine in the chloroplast envelope after import of lysophosphatidylcholine from endoplasmic reticulum membranes. Eur. J. Biochem. 228, 490-497. doi: 10. 1111/j.1432-1033.1995.tb20288.x and Michaud, 2018). These stress-activated modifications constitute powerful tools to identify candidates and to dissect how contact sites are formed, how lipids are transferred and how these processes are regulated in cells. Most of the proteins identified in other organisms, such as the different families of LTPs (i.e., E-Syt, ORP) and VAP, are also conserved in plants. Interestingly, the number of homologs of these proteins are generally higher in plants than in yeast and mammals, and most of them are overexpressed during stress (Umate, 2011; Perez-Sancho et al., 2016; Wang et al., 2016). This highlights the complexity of non-vesicular lipid transport in plants and open important perspectives of investigation in this field for the next decades.

\section{AUTHOR CONTRIBUTIONS}

All authors contributed to the writing of the review. MM created the figures.

\section{FUNDING}

This work was supported by the French National Research Agency (GlycoAlps Project No. ANR-15-IDEX-02 and ANR-10LABX-49-01 GRAL).

\section{ACKNOWLEDGMENTS}

We thank Fabrice Rébeillé and Melissa Conte for the critical reading of the manuscript.

Block, M. A., and Jouhet, J. (2015). Lipid trafficking at endoplasmic reticulumchloroplast membrane contact sites. Curr. Opin. Cell Biol. 35, 21-29. doi: 10. 1016/j.ceb.2015.03.004

Botella, C., Sautron, E., Boudiere, L., Michaud, M., Dubots, E., YamaryoBotte, Y., et al. (2016). ALA10, a phospholipid flippase, controls FAD2/FAD3 desaturation of phosphatidylcholine in the er and affects chloroplast lipid composition in Arabidopsis thaliana. Plant Physiol. 170, 1300-1314. doi: 10. 1104/pp.15.01557

Boudiere, L., Botte, C. Y., Saidani, N., Lajoie, M., Marion, J., Brehelin, L., et al. (2012). Galvestine-1, a novel chemical probe for the study of the glycerolipid homeostasis system in plant cells. Mol. Biosyst. 8, 2023-2035. doi: 10.1039/ c2mb25067e

Cai, Y., Goodman, J. M., Pyc, M., Mullen, R. T., Dyer, J. M., and Chapman, K. D. (2015). Arabidopsis SEIPIN proteins modulate triacylglycerol accumulation and influence lipid droplet proliferation. Plant Cell 27, 2616-2636. doi: 10.1105/ tpc. 15.00588

Chang, C. L., Chen, Y. J., and Liou, J. (2017). ER-plasma membrane junctions: why and how do we study them? Biochim. Biophys. Acta 1864, 1494-1506. doi: 10.1016/j.bbamcr.2017.05.018

Chou, M. X., Wei, X. Y., Chen, D. S., and Zhou, J. C. (2006). Thirteen nodulespecific or nodule-enhanced genes encoding products homologous to cysteine cluster proteins or plant lipid transfer proteins are identified in Astragalus sinicus L. by suppressive subtractive hybridization. J. Exp. Bot. 57, 2673-2685. doi: 10.1093/jxb/er1030

Cohen, S., Valm, A. M., and Lippincott-Schwartz, J. (2018). Interacting organelles. Curr. Opin. Cell Biol. 53, 84-91. doi: 10.1016/j.ceb.2018.06.003

Cui, S., Hayashi, Y., Otomo, M., Mano, S., Oikawa, K., Hayashi, M., et al. (2016). Sucrose production mediated by lipid metabolism suppresses the physical 
interaction of peroxisomes and oil bodies during germination of Arabidopsis thaliana. J. Biol. Chem. 291, 19734-19745. doi: 10.1074/jbc.M116.748814

Dormann, P., and Benning, C. (2002). Galactolipids rule in seed plants. Trends Plant Sci. 7, 112-118. doi: 10.1016/S1360-1385(01)02216-6

Downie, J. A. (2014). Legume nodulation. Curr. Biol. 24, R184-R190. doi: 10.1016/ j.cub.2014.01.028

Dubots, E., Audry, M., Yamaryo, Y., Bastien, O., Ohta, H., Breton, C., et al. (2010). Activation of the chloroplast monogalactosyldiacylglycerol synthase MGD1 by phosphatidic acid and phosphatidylglycerol. J. Biol. Chem. 285, 6003-6011. doi: 10.1074/jbc.M109.071928

Eastmond, P. J. (2006). SUGAR-DEPENDENT1 encodes a patatin domain triacylglycerol lipase that initiates storage oil breakdown in germinating Arabidopsis seeds. Plant Cell 18, 665-675. doi: 10.1105/tpc.105.040543

Eckardt, N. A. (2008). Arabidopsis synaptotagmin1 maintains plasma membrane integrity. Plant Cell 20, 3182. doi: 10.1105/tpc.108.201211

Fan, J., and $\mathrm{Xu}, \mathrm{C}$. (2011). Genetic analysis of arabidopsis mutants impaired in plastid lipid import reveals a role of membrane lipids in chloroplast division. Plant Signal. Behav. 6, 458-460. doi: 10.4161/psb.6.3.14715

Gao, Q., and Goodman, J. M. (2015). The lipid droplet-a well-connected organelle. Front. Cell Dev. Biol. 3:49. doi: 10.3389/fcell.2015.00049

Gaude, N., Brehelin, C., Tischendorf, G., Kessler, F., and Dormann, P. (2007). Nitrogen deficiency in Arabidopsis affects galactolipid composition and gene expression and results in accumulation of fatty acid phytyl esters. Plant J. Cell Mol. Biol. 49, 729-739. doi: 10.1111/j.1365-313X.2006.02992.x

Gaude, N., Nakamura, Y., Scheible, W. R., Ohta, H., and Dormann, P. (2008). Phospholipase C5 (NPC5) is involved in galactolipid accumulation during phosphate limitation in leaves of Arabidopsis. Plant J. 56, 28-39. doi: 10.1111/J. 1365-313x.2008.03582.X

Gaude, N., Tippmann, H., Flemetakis, E., Katinakis, P., Udvardi, M., and Dormann, P. (2004). The galactolipid digalactosyldiacylglycerol accumulates in the peribacteroid membrane of nitrogen-fixing nodules of soybean and Lotus. J. Biol. Chem. 279, 34624-34630. doi: 10.1074/Jbc.M404098200

Gavrin, A., Kulikova, O., Bisseling, T., and Fedorova, E. E. (2017). Interface symbiotic membrane formation in root nodules of Medicago truncatula: the role of synaptotagmins MtSyt1, MtSyt2 and MtSyt3. Front. Plant Sci. 8:201. doi: 10.3389/fpls.2017.00201

Hanson, M. R., and Sattarzadeh, A. (2011). Stromules: recent insights into a long neglected feature of plastid morphology and function. Plant Physiol. 155, 1486-1492. doi: 10.1104/pp.110.170852

Harrison, M. J., and Ivanov, S. (2017). Exocytosis for endosymbiosis: membrane trafficking pathways for development of symbiotic membrane compartments. Curr. Opin. Plant Biol. 38, 101-108. doi: 10.1016/j.pbi.2017.04.019

Hartel, H., Dormann, P., and Benning, C. (2000). DGD1-independent biosynthesis of extraplastidic galactolipids after phosphate deprivation in Arabidopsis. Proc. Natl. Acad. Sci. U.S.A. 97, 10649-10654. doi: 10.1073/pnas.1803 20497

Hartel, H., Dormann, P., and Benning, C. (2001). Galactolipids not associated with the photosynthetic apparatus in phosphate-deprived plants. J. Photochem. Photobiol. B Biol. 61, 46-51. doi: 10.1016/S1011-1344(01)00144-0

Hayashi, M., Nito, K., Takei-Hoshi, R., Yagi, M., Kondo, M., Suenaga, A., et al. (2002). Ped3p is a peroxisomal ATP-binding cassette transporter that might supply substrates for fatty acid beta-oxidation. Plant Cell Physiol. 43, 1-11. doi: $10.1093 / \mathrm{pcp} / \mathrm{pcf0} 23$

Hsueh, Y. C., Ehmann, C., Flinner, N., Ladig, R., and Schleiff, E. (2017). The plastid outer membrane localized LPTD1 is important for glycerolipid remodeling under phosphate starvation. Plant Cell Environ. 40, 1643-1657. doi: 10.1111/ pce. 12973

Huang, A. H. C. (2018). Plant lipid droplets and their associated proteins: potential for rapid advances. Plant Physiol. 176, 1894-1918. doi: 10.1104/pp.17.01677

Hurlock, A. K., Roston, R. L., Wang, K., and Benning, C. (2014). Lipid trafficking in plant cells. Traffic 15, 915-932. doi: 10.1111/tra.12187

Jessen, D., Roth, C., Wiermer, M., and Fulda, M. (2015). Two activities of longchain acyl-coenzyme a synthetase are involved in lipid trafficking between the endoplasmic reticulum and the plastid in Arabidopsis. Plant Physiol. 167, 351-366. doi: 10.1104/pp.114.250365

Jouhet, J., Marechal, E., Baldan, B., Bligny, R., Joyard, J., and Block, M. A. (2004). Phosphate deprivation induces transfer of DGDG galactolipid from chloroplast to mitochondria. J. Cell Biol. 167, 863-874. doi: 10.1083/jcb.200407022
Jouhet, J., Marechal, E., Bligny, R., Joyard, J., and Block, M. A. (2003). Transient increase of phosphatidylcholine in plant cells in response to phosphate deprivation. FEBS Lett. 544, 63-68. doi: 10.1016/S0014-59793(03)00 477-0

Jurgens, G. (2004). Membrane trafficking in plants. Annu. Rev. Cell Dev. Biol. 20, 481-504. doi: 10.1146/annurev.cellbio.20.082503.103057

Kelly, A. A., Kalisch, B., Holzl, G., Schulze, S., Thiele, J., Melzer, M., et al. (2016). Synthesis and transfer of galactolipids in the chloroplast envelope membranes of Arabidopsis thaliana. Proc. Natl. Acad. Sci. U.S.A. 113, 10714-10719. doi: 10.1073/pnas.1609184113

Kim, H., Kwon, H., Kim, S., Kim, M. K., Botella, M. A., Yun, H. S., et al. (2016). Synaptotagmin 1 negatively controls the two distinct immune secretory pathways to powdery mildew fungi in Arabidopsis. Plant Cell Physiol. 57, 1133-1141. doi: 10.1093/pcp/pcw061

Kohler, R. H., and Hanson, M. R. (2000). Plastid tubules of higher plants are tissue-specific and developmentally regulated. J. Cell Sci. 113( Pt 1), 81-89.

Krahmer, N., Guo, Y., Wilfling, F., Hilger, M., Lingrell, S., Heger, K., et al. (2011). Phosphatidylcholine synthesis for lipid droplet expansion is mediated by localized activation of CTP:phosphocholine cytidylyltransferase. Cell Metab. 14, 504-515. doi: 10.1016/j.cmet.2011.07.013

Krenz, B., Guo, T. W., and Kleinow, T. (2014). Stromuling when stressed! Acta Soc. Bot. Pol. 83, 325-329. doi: 10.5586/asbp.2014.050

LaBrant, E., Barnes, A. C., and Roston, R. L. (2018). Lipid transport required to make lipids of photosynthetic membranes. Photosynth. Res. 138, 345-360. doi: 10.1007/s11120-018-0545-5

Larsson, K. E., Kjellberg, J. M., Tjellstrom, H., and Sandelius, A. S. (2007). LysoPC acyltransferase/PC transacylase activities in plant plasma membrane and plasma membrane-associated endoplasmic reticulum. BMC Plant Biol. 7:64. doi: 10.1186/1471-2229-7-64

Lei, L., Chen, L., Shi, X., Li, Y., Wang, J., Chen, D., et al. (2014). A nodule-specific lipid transfer protein AsE246 participates in transport of plant-synthesized lipids to symbiosome membrane and is essential for nodule organogenesis in Chinese milk vetch. Plant Physiol. 164, 1045-1058. doi: 10.1104/pp.113. 232637

Levy, A., Zheng, J. Y., and Lazarowitz, S. G. (2015). Synaptotagmin SYTA forms ER-plasma membrane junctions that are recruited to plasmodesmata for plant virus movement. Cur. Biol. 25, 2018-2025. doi: 10.1016/j.cub.2015.06.015

Lewis, J. D., and Lazarowitz, S. G. (2010). Arabidopsis synaptotagmin SYTA regulates endocytosis and virus movement protein cell-to-cell transport. Proc. Natl. Acad. Sci. U.S.A. 107, 2491-2496. doi: 10.1073/pnas.0909080107

Li, Z., Gao, J., Benning, C., and Sharkey, T. D. (2012). Characterization of photosynthesis in Arabidopsis ER-to-plastid lipid trafficking mutants. Photosyn. Res. 112, 49-61. doi: 10.1007/s11120-012-9734-9

Li-Beisson, Y., Neunzig, J., Lee, Y., and Philippar, K. (2017). Plant membraneprotein mediated intracellular traffic of fatty acids and acyl lipids. Curr. Opin. Plant Biol. 40, 138-146. doi: 10.1016/j.pbi.2017.09.006

Li-Beisson, Y., Shorrosh, B., Beisson, F., Andersson, M. X., Arondel, V., Bates, P. D., et al. (2013). Acyl-lipids metabolism. Arabibopsis Book 8:e0133. doi: $10.1199 /$ tab.0161

Marechal, E., and Bastien, O. (2014). Modeling of regulatory loops controlling galactolipid biosynthesis in the inner envelope membrane of chloroplasts. J. Theor. Biol. 361, 1-13. doi: 10.1016/j.jtbi.2014.07.013

McFarlane, H. E., Lee, E. K., van Bezouwen, L. S., Ross, B., Rosado, A., and Samuels, A. L. (2017). Multiscale structural analysis of plant ER-PM contact sites. Plant Cell Physiol. 58, 478-484. doi: 10.1093/pcp/pcw224

Mei, C. E., Cussac, M., Haslam, R. P., Beaudoin, F., Wong, Y. S., Marechal, E., et al. (2017). C1 metabolism inhibition and nitrogen deprivation trigger triacylglycerol accumulation in arabidopsis thaliana cell cultures and highlight a role of NPC in phosphatidylcholine-to-triacylglycerol pathway. Front. Plant Sci. 7:2014. doi: 10.3389/fpls.2016.02014

Michaud, M., Gros, V., Tardif, M., Brugiere, S., Ferro, M., Prinz, W. A., et al. (2016). AtMic60 is involved in plant mitochondria lipid trafficking and is part of a large complex. Curr. Biol. 26, 627-639. doi: 10.1016/j.cub.2016.01.011

Michaud, M., Prinz, W. A., and Jouhet, J. (2017). Glycerolipid synthesis and lipid trafficking in plant mitochondria. FEBS J. 284, 376-390. doi: 10.1111/febs.13812

Moellering, E. R., and Benning, C. (2011). Galactoglycerolipid metabolism under stress: a time for remodeling. Trends Plant Sci. 16, 98-107. doi: 10.1016/j.tplants. 2010.11.004 
Mongrand, S., Bessoule, J. J., Cabantous, F., and Cassagne, C. (1998). The C-16:3/C-18: 3 fatty acid balance in photosynthetic tissues from 468 plant species. Phytochemistry 49, 1049-1064. doi: 10.1016/S0031-9422(98)00 243-X

Mongrand, S., Cassagne, C., and Bessoule, J. J. (2000). Import of lysophosphatidylcholine into chloroplasts likely at the origin of eukaryotic plastidial lipids. Plant Physiol. 122, 845-852. doi: 10.1104/pp.122.3.845

Moreau, P., Bessoule, J. J., Mongrand, S., Testet, E., Vincent, P., and Cassagne, C. (1998). Lipid trafficking in plant cells. Prog. Lipid Res. 37, 371-391. doi: 10.1016/ S0163-7827(98)00016-2

Mueller-Schuessele, S. J., and Michaud, M. (2018). Plastid transient and stable interactions with other cell compartments. Methods Mol. Biol. 1829, 87-109. doi: 10.1007/978-1-4939-8654-5_6

Murphy, S. E., and Levine, T. P. (2016). VAP, a versatile access point for the endoplasmic reticulum: review and analysis of FFAT-like motifs in the VAPome. Biochim. Biophys. Acta 1861(8 Pt B), 952-961. doi: 10.1016/j.bbalip.2016.02.009

Olzmann, J. A., and Carvalho, P. (2018). Dynamics and functions of lipid droplets. Nat. Rev. Mol. Cell Biol. doi: 10.1038/s41580-018-0085-z [Epub ahead of print.,

Pant, B. D., Burgos, A., Pant, P., Cuadros-Inostroza, A., Willmitzer, L., and Scheible, W. R. (2015). The transcription factor PHR1 regulates lipid remodeling and triacylglycerol accumulation in Arabidopsis thaliana during phosphorus starvation. J. Exp. Bot. 66, 1907-1918. doi: 10.1093/jxb/eru535

Perez-Sancho, J., Tilsner, J., Samuels, A. L., Botella, M. A., Bayer, E. M., and Rosado, A. (2016). Stitching organelles: organization and function of specialized membrane contact sites in plants. Trends Cell Biol. 26, 705-717. doi: 10.1016/j. tcb.2016.05.007

Perez-Sancho, J., Vanneste, S., Lee, E., McFarlane, H. E., Esteban Del Valle, A., Valpuesta, V., et al. (2015). The Arabidopsis synaptotagmin1 is enriched in endoplasmic reticulum-plasma membrane contact sites and confers cellular resistance to mechanical stresses. Plant Physiol. 168, 132-143. doi: 10.1104/pp. 15.00260

Phillips, M. C., Johnson, W. J., and Rothblat, G. H. (1987). Mechanisms and consequences of cellular cholesterol exchange end transfer. Biochim. Biophys. Acta 906, 223-276. doi: 10.1016/0304-4157(87)90013-X

Phillips, M. J., and Voeltz, G. K. (2016). Structure and function of ER membrane contact sites with other organelles. Nat. Rev. Mol. Cell Biol. 17, 69-82. doi: $10.1038 / \mathrm{nrm} .2015 .8$

Poirier, Y., Thoma, S., Somerville, C., and Schiefelbein, J. (1991). Mutant of Arabidopsis deficient in xylem loading of phosphate. Plant Physiol. 97, 1087-1093. doi: 10.1104/pp.97.3.1087

Poulsen, L. R., Lopez-Marques, R. L., Pedas, P. R., McDowell, S. C., Brown, E., Kunze, R., et al. (2015). A phospholipid uptake system in the model plant Arabidopsis thaliana. Nat. Commun. 6:7649. doi: 10.1038/ncomms 8649

Pyc, M., Cai, Y., Greer, M. S., Yurchenko, O., Chapman, K. D., Dyer, J. M., et al. (2017). Turning over a new leaf in lipid droplet biology. Trends Plant Sci. 22, 596-609. doi: 10.1016/j.tplants.2017.03.012

Renaudin, S., and Capdepon, M. (1977). Association of endoplasmic-reticulum and plastids in tozzia-alpina 1 scale leaves. J. Ultrastruct. Res. 61, 303-308. doi: 10.1016/S0022-5320(77)80055-5

Roston, R., Gao, J., Xu, C., and Benning, C. (2011). Arabidopsis chloroplast lipid transport protein TGD2 disrupts membranes and is part of a large complex. Plant J. Cell Mol. Biol. 66, 759-769. doi: 10.1111/j.1365-313X.2011.04536.x

Saheki, Y., and De Camilli, P. (2017a). Endoplasmic reticulum-plasma membrane contact sites. Annu. Rev. Biochem. 86, 659-684. doi: 10.1146/annurev-biochem061516-044932

Saheki, Y., and De Camilli, P. (2017b). The extended-synaptotagmins. Biochim. Biophys. Acta 1864, 1490-1493. doi: 10.1016/j.bbamcr.2017.03.013

Salo, V. T., Belevich, I., Li, S., Karhinen, L., Vihinen, H., Vigouroux, C., et al. (2016). Seipin regulates ER-lipid droplet contacts and cargo delivery. EMBO J. 35, 2699-2716. doi: 10.15252/embj.201695170

Sandelius, A. S., and Liljenberg, C. (1982). Light-induced-changes in the lipidcomposition and ultrastructure of plastids from potato-tubers. Physiol. Plant. 56, 266-272. doi: 10.1111/j.1399-3054.1982.tb00337.x

Saravanan, R. S., Slabaugh, E., Singh, V. R., Lapidus, L. J., Haas, T., and Brandizzi, F. (2009). The targeting of the oxysterol-binding protein ORP3a to the endoplasmic reticulum relies on the plant VAP33 homolog PVA12. Plant J. 58, 817-830. doi: 10.1111/j.1365-313X.2009.03815.x
Schapire, A. L., Voigt, B., Jasik, J., Rosado, A., Lopez-Cobollo, R., Menzel, D., et al. (2008). Arabidopsis synaptotagmin 1 is required for the maintenance of plasma membrane integrity and cell viability. Plant Cell 20, 3374-3388. doi: 10.1105/tpc.108.063859

Schattat, M., Barton, K., and Mathur, J. (2011). Correlated behavior implicates stromules in increasing the interactive surface between plastids and ER tubules. Plant Signal. Behav. 6, 715-718. doi: 10.4161/psb.6.5.15085

Shimojima, M., Madoka, Y., Fujiwara, R., Murakawa, M., Yoshitake, Y., Ikeda, K., et al. (2015). An engineered lipid remodeling system using a galactolipid synthase promoter during phosphate starvation enhances oil accumulation in plants. Front. Plant Sci. 6:664. doi: 10.3389/fpls.2015.00664

Siao, W., Wang, P., Voigt, B., Hussey, P. J., and Baluska, F. (2016). Arabidopsis SYT1 maintains stability of cortical endoplasmic reticulum networks and VAP27-1-enriched endoplasmic reticulum-plasma membrane contact sites. J. Exp. Bot. 67, 6161-6171. doi: 10.1093/jxb/erw381

Somerharju, P. (2015). Is spontaneous translocation of polar lipids between cellular organelles negligible? Lipid Insights 8(Suppl. 1), 87-93. doi: 10.4137/LPI.S3 1616

Tan, X., Wang, Q., Tian, B., Zhang, H., Lu, D., and Zhou, J. (2011). A Brassica napus lipase locates at the membrane contact sites involved in chloroplast development. PLoS One 6:e26831. doi: 10.1371/journal.pone.002 6831

Thazar-Poulot, N., Miquel, M., Fobis-Loisy, I., and Gaude, T. (2015). Peroxisome extensions deliver the Arabidopsis SDP1 lipase to oil bodies. Proc. Natl. Acad. Sci. U.S.A. 112, 4158-4163. doi: 10.1073/pnas.1403322112

Udvardi, M., and Poole, P. S. (2013). Transport and metabolism in legume-rhizobia symbioses. Annu. Rev. Plant Biol. 64, 781-805. doi: 10.1146/annurev-arplant050312-120235

Umate, P. (2011). Oxysterol binding proteins (OSBPs) and their encoding genes in Arabidopsis and rice. Steroids 76, 524-529. doi: 10.1016/j.steroids.2011.01.007

Wang, P., Hawes, C., and Hussey, P. J. (2017). Plant endoplasmic reticulum-plasma membrane contact sites. Trends Plant Sci. 22, 289-297. doi: 10.1016/j.tplants. 2016.11.008

Wang, P., Hawkins, T. J., Richardson, C., Cummins, I., Deeks, M. J., Sparkes, I., et al. (2014). The plant cytoskeleton, NET3C, and VAP27 mediate the link between the plasma membrane and endoplasmic reticulum. Cur. Biol. 24, 1397-1405. doi: 10.1016/j.cub.2014.05.003

Wang, P., Richardson, C., Hawkins, T. J., Sparkes, I., Hawes, C., and Hussey, P. J. (2016). Plant VAP27 proteins: domain characterization, intracellular localization and role in plant development. New Phytol. 210, 1311-1326. doi: $10.1111 /$ nph.13857

Wang, Z., Xu, C., and Benning, C. (2012). TGD4 involved in endoplasmic reticulum-to-chloroplast lipid trafficking is a phosphatidic acid binding protein. Plant J. Cell Mol. Biol. 70, 614-623. doi: 10.1111/j.1365-313X.2012. 04900.x

Whitehead, L. F., and Day, D. A. (1997). The peribacteroid membrane. Physiol. Plant 100, 30-44. doi: 10.1111/j.1399-3054.1997.tb03452.x

Wilfling, F., Wang, H., Haas, J. T., Krahmer, N., Gould, T. J., Uchida, A., et al. (2013). Triacylglycerol synthesis enzymes mediate lipid droplet growth by relocalizing from the ER to lipid droplets. Dev. Cell 24, 384-399. doi: 10.1016/j. devcel.2013.01.013

Winter, D., Vinegar, B., Nahal, H., Ammar, R., Wilson, G. V., and Provart, N. J. (2007). An "Electronic Fluorescent Pictograph" browser for exploring and analyzing large-scale biological data sets. PLoS One 2:e718. doi: 10.1371/journal. pone. 0000718

Wong, L. H., Copic, A., and Levine, T. P. (2017). Advances on the transfer of lipids by lipid transfer proteins. Trends Biochem. Sci. 42, 516-530. doi: 10.1016/j.tibs. 2017.05.001

Wu, H., Carvalho, P., and Voeltz, G. K. (2018). Here, there, and everywhere: the importance of ER membrane contact sites. Science 361:6401. doi: 10.1126/ science.aan5835

Xu, C. C., Fan, J., Froehlich, J. E., Awai, K., and Benning, C. (2005). Mutation of the TGD1 chloroplast envelope protein affects phosphatidate metabolism in Arabidopsis. Plant Cell 17, 3094-3110. doi: 10.1105/Tpc.105.035592

Yamaryo, Y., Dubots, E., Albrieux, C., Baldan, B., and Block, M. A. (2008) Phosphate availability affects the tonoplast localization of PLD zeta 2, an Arabidopsis thaliana phospholipase D. FEBS Lett. 582, 685-690. doi: 10.1016/ J.Febslet.2008.01.039 
Yamazaki, T., Kawamura, Y., Minami, A., and Uemura, M. (2008). Calciumdependent freezing tolerance in Arabidopsis involves membrane resealing via synaptotagmin SYT1. Plant Cell 20, 3389-3404. doi: 10.1105/tpc.108. 062679

Yang, Y., and Benning, C. (2018). Functions of triacylglycerols during plant development and stress. Curr. Opin. Biotechnol. 49, 191-198. doi: 10.1016/j. copbio.2017.09.003

Yang, Y., Yu, X., Song, L., and An, C. (2011). ABI4 activates DGAT1 expression in Arabidopsis seedlings during nitrogen deficiency. Plant Physiol. 156, 873-883. doi: 10.1104/pp.111.175950

Yin, C., Andersson, M. X., Zhang, H., and Aronsson, H. (2015). Phosphatidylcholine is transferred from chemically-defined liposomes to chloroplasts through proteins of the chloroplast outer envelope membrane. FEBS Lett. 589, 177-181. doi: 10.1016/j.febslet.2014.11.044
Zheng, G., Li, L., and Li, W. (2016). Glycerolipidome responses to freezing- and chilling-induced injuries: examples in Arabidopsis and rice. BMC Plant Biol. 16:70. doi: 10.1186/s12870-016-0758-8

Conflict of Interest Statement: The authors declare that the research was conducted in the absence of any commercial or financial relationships that could be construed as a potential conflict of interest.

Copyright (c) 2019 Michaud and Jouhet. This is an open-access article distributed under the terms of the Creative Commons Attribution License (CC BY). The use, distribution or reproduction in other forums is permitted, provided the original author(s) and the copyright owner(s) are credited and that the original publication in this journal is cited, in accordance with accepted academic practice. No use, distribution or reproduction is permitted which does not comply with these terms. 